\title{
A CASE OF GLYCOGEN STORAGE DISEASE TYPE 1a MIMICKING FAMILIAL CHYLOMICRONEMIA SYNDROME
}

\author{
Olgac A ${ }^{1},{ }^{*}$, Okur İ², Biberoğlu G², Ezgü FS², Tümer L²
}

*Corresponding Author: Dr. Asburce Olgac, Department of Pediatric Metabolism, University of Health Sciences, Dr. Sami Ulus Maternity and Child Health, Training and Research Hospital, Ankara, Turkey. Tel.: +90-312-305-600. Fax:+90-312-317-03-53. E-mail: mabolgac@yahoo.com

\begin{abstract}
Glycogen storage disease type 1a (GSD1a) is an autosomal recessively inherited inborn error of metabolism caused by a mutation in the G6PC gene, which encodes the catalytic subunit of glucose- 6 -phosphatase- $\alpha$ (G6Pase- $\alpha$ ) enzyme. This enzyme plays a role in the final step of gluconeogenesis and glycogenolysis. Patients carrying GSD1a show growth retardation, hypoglycemia, hepatomegaly, hepatic steatosis, hyperlipidemia, hyperuricemia and lactic acidemia. Long-term symptoms include gouty arthritis and uric acid stones, osteoporosis, renal failure, intestinal impairment, cirrhosis and hepatic adenomas, and eventually, hepatocellular carcinoma. Hyperlipidemia is the indicator of poor metabolic control in GSD1a. Patients with variable levels of triglycerides (TGs) have been reported in the literature. We present a case of GSD1a that presented with severe hypertriglyceridemia (HTG) mimicking familial chylomicronemia syndrome.
\end{abstract}

Keywords: Familial chylomicronemia syndrome (FCS); Glycogen storage disease type 1a (GSD1a); Hypertriglyceridemia (HTG); Pancreatitis.

\section{INTRODUCTION}

Glycogen storage disease type 1a (GSD1a) (Von Gierke disease) is an autosomal recessively inherited inborn error of metabolism caused by a mutation in the G6PC gene, which encodes the catalytic subunit of glu-cose-6phosphatase- $\alpha$ (G6Pase- $\alpha$ ) enzyme. This enzyme plays a role in the final step of gluconeogenesis and glyco-genolysis

\footnotetext{
${ }^{1}$ Department of Pediatric Metabolism, Division of Pediatric Metabolism, University of Health Sciences, Dr. Sami Ulus Maternity and Child Health, Training and Research Hospital, Ankara, Turkey

2 Department of Pediatric Metabolism and Nutrition, Gazi University Hospital, Ankara, Turkey
}

by hydrolyzing G6P to glucose and phosphate. Due to the deficient activity of this enzyme, excessive accumulation of glycogen occurs in the liver, kidney, skeletal muscles and intestinal mucosa. As glucose homeostasis cannot be maintained during fasting, life-threatening hypoglycemia episodes may be seen [1].

Hyperlipidemia is the indicator of poor metabolic control in GSD1a [2]. Patients with variable levels of triglycerides (TGs) have been reported in the literature. We present a case of GSD1a that was referred due to severe hypertriglyceridemia (HTG) mimicking familial chylomicronemia syndrome (FCS). Written consent was obtained from parents for the preparation and publication of this manuscript.

Patients and Methods. The case being reported here is a 2-month-old female infant, an offspring of a non consanguineous marriage. She was born at term after an uncomplicated pregnancy, and hypoglycemia was detected once in the second postnatal hour, that was easily resolved with oral dextrose and did not recur. She was fed exclusively with breast-milk with frequent feeds, and her weight gain was adequate afterwards. When she was 2 months old, she had an episode of irritability, fever and vomiting. She was admitted to an external clinic, where laboratory analyses including complete blood count, biochemistry and acute phase reactants were performed that showed normal results. Urinalysis was negative. Since the medical staff noticed her blood to be lipemic during blood draw, the lipid profile was checked showing severely elevated triglyceride (TG) levels $(18,000.0 \mathrm{mg} / \mathrm{dL}$, normal range: $2.0-150.0 \mathrm{mg} / \mathrm{dL}$ ). Heparinized dextrose infusion was initiated, and she was referred to our clinic with a pre diagnosis of FCS.

Upon admission, she was in good condition. Her weight, height and head circumference were within normal centiles. Physical examination revealed a rounded doll's face, abdominal distension, and hepatomegaly (liver was 8 $\mathrm{cm}$ palpable at the mid-clavicular margin). The spleen was 
not palpable. Lipemia was noticed (Figure-1). Laboratory analyses showed elevated TG $(10,000.0 \mathrm{mg} / \mathrm{dL})$, total cholesterol: $927.0 \mathrm{mg} / \mathrm{dL}$ (normal range: $5.0-120.0 \mathrm{mg} / \mathrm{dL}$ ), and lactate: $6.0 \mathrm{mg} / \mathrm{dL}$ (normal range: $0.2 .0-2.0 \mathrm{mg} / \mathrm{dL}$ ). Low-density lipoprotein (LDL) levels could not be evaluated due to high levels of TGs. Other biochemical analyses including kidney and liver function tests, uric acid, amilase, lipase and blood-gas analysis were within normal ranges.

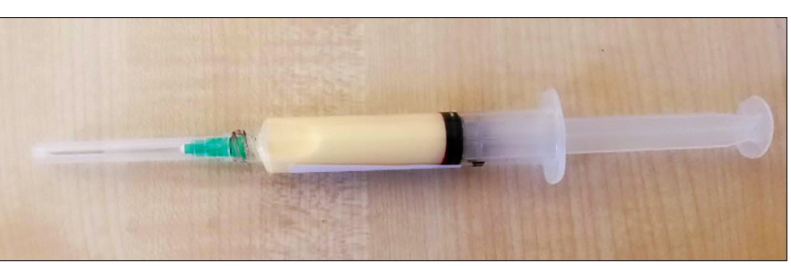

Figure 1. Milky lipemic appearance of the patient's blood due to hyperlipidemia.

Due to the initial diagnosis of familial hyperlipidemia, hydrolyzed formula containing 50.0\% medium-chain triglycerides (MCTs) and heparin with dextrose infusion was continued. Glucose infusion rate was increased due to hypoglycemia $(47.0 \mathrm{mg} / \mathrm{dL})$. Insulin level was checked during hypoglycemia and was found to be low. Urine ketone levels were positive. Metabolic tests including acylcarnitinites, plasma and urine amino acids, and biotinidase level were inconclusive. Highly increased lactic, 3-hydroxybutiric and acetoacetic acids were detected in urine organic acid analysis. Urine was checked for the presence reducing substance, that was found to be negative. Triglyceride levels decreased gradually to $1000.0 \mathrm{mg} / \mathrm{dL}$ and heparin infusion was terminated. Total cholesterol (TC) levels fell to within reference ranges. Abdominal ultra-sonography revealed increased diffuse echogenity of the liver.

Due to hypoglycemia and ketonuria, a gluconeogenesis disorder was suspected. In the context of clinical and laboratory findings, GSD1a was the most probable diagnosis. Molecular genetic analysis of the G6PC gene showed a homozygous missense R83C mutation that was previously reported to be pathogenic. Frequent feedings with formula containing MCTs was continued and TG levels ranged within 300.0-400.0 mg/dL despite compliance to diet. The patient is now 6 years old and severe TG elevation has not recurred.

\section{DISCUSSION}

HTG in childhood may be due to genetic factors or secondary to several conditions (e.g., metabolic syndrome, drugs, inborn errors of metabolism). Severe HTG, in which fasting plasma TG levels are above $885.0 \mathrm{mg} / \mathrm{dL}$ $(10.0 \mathrm{mmol} / \mathrm{L})$, is usually due to high levels of intestinally derived chylomicron particles (TG-rich lipoproteins). In pediatric patients, this condition is mainly due to endothelial-bound lipoprotein lipase (LPL) deficiency, which is an autosomal recessive disorder affecting the catabolism of chylomicrons, or its coactivators. It is also called FCS, and occurs due to biallelic mutations of several genes [2]. Triglyceride levels are variable in FCS, but may rise up to 100 -fold, and the diagnosis should be made early to prevent acute pancreatitis due to HTG. Although hypoglycemia is not a direct symptom of FCS, pancreatic involvement due to HTG may cause distruption of glucose homeostasis [3]. Treatment includes dietary fat restriction $(<15.0 \%$ of daily caloric intake), avoidance of high glycemic index foods, supplementation with omega-3 and medium-chain TGs. Fibrates and niacin may be used that are somewhat effective [1].

Several patients with FCS diagnosed in the neonatal period have been reported in the literature $[2,4,5]$. Shah et al. [2] have reported a 23-day-old baby with confirmed LPL deficiency and acute pancreatitis, who was admitted due to fever, vomiting, lethargy and lipemia (elevated TG levels up to $10,300 \mathrm{mg} / \mathrm{dL}$ ), similar to the initial presentation of our patient.

Hypertriglyceridemia may also occur due to glycogen storage diseases, of which GSD1a is the most frequent, and the incidence is estimated to be $1 / 100,000$ births [1]. The disease occurs due to the defects on the G6PC gene, which is located on chromosome 17q21.31. The G6PC gene encodes the catalytic subunit of the enzyme G6Pase- $\alpha$ that is responsible for the last step of glycogenolysis [6]. As a result of the deficient activity of this enzyme, the patients are unable to tolerate short-term fasting (2-4 hours) and hypoglycemia occurs. Disturbed glucose homeostasis, leads to secondary metabolic derangement causing hyperlipidemia and hepatosteatosis due to TG accumulation in the liver. Patients display lactic acidosis, a protruded abdomen due to hepatomegaly, anemia and bleeding tendency due to platelet dysfunction, a round doll-like face, muscle weakness, osteopenia and short stature [1].

Hyperlipidemia is a frequently encountered symptom that usually involves TGs and cholesterol. Talente et al. [7] reported HTG to be $100.0 \%$ in adults with GSD1a. Hypertriglyceridemia and hypercholesterolemia (HC) mainly occur due to poor metabolic control and sustained hypoglycemia that causes lipid synthesis in the liver. Although the exact mechanism is unknown, increased lipidogenesis and decreased clearance and uptake of lipids due to the deficient activity of lipoprotein lipase and hepatic lipase, are the suggested factors that increase blood TG and TC levels. Excessive intake of carbohydrates can also increase lipid synthesis in the liver, and the activation of lipidogenic genes due to the accumulation of glucose- 
6-phosphatase (G6P), could be another probability. Since insulin levels are low in GSD1a patients, increased very low-density lipoprotein (VLDL) levels that are rich in TGs, may be another contributing factor. Blood TG and TC levels are variable among cases, and during the followup period, even in the same patient. Patients with poorly managed GSD1a sometimes develop variable levels of HTG that is a significant risk factor for acute pancreatitis, especially if above $1000.0 \mathrm{mg} / \mathrm{dL}$ [8].

Treatment is mainly diet therapy with frequent feedings to prevent hypoglycemia and elimination of lactose and fructose from diet. Patients may require continuous nocturnal gastric drip feeding. Uncooked cornstarch and maltodextrin can be used after the age of 1 to prevent hypoglycemia. Unfortunately, hyperlipidemia does not completely resolve with dietary therapy [9]. Medium-chain TGs are known to have beneficial effects on TG levels, as they are directly absorbed from the portal vein. Longterm complications, including liver failure, are also not prevented by dietary treatment. Liver transplantation is only indicated in patients with hepatic malignancies that are unresectable or do not benefit from dietary therapy [10].

\section{CONCLUSIONS}

Our case reveals that the diagnosis of GSD1a may be challenging, due to its variable presentations. Although hypoglycemia is a key symptom, it may be overlooked and lead to delay of diagnosis. Symptoms of GSD1 a should be kept in mind and investigated in patients with metabolic decompansation signs including hepatomegaly, hepatosteatosis and dyslipidemia.

Declaration of Interest. The authors report no conflicts of interest. The authors alone are responsible for the content and writing of this article.

\section{REFERENCES}

1. Chou JY, Kim GY, Cho JH. Recent development and gene therapy for glycogen storage disease type Ia. Liver Res. 2017;1(3): 174-180.

2. Shah MH, Roshan R, Desai R, Kadam SS. Neonatal hyperlipidemia with pancreatitis: novel gene mutation of lipoprotein lipase. J Postgrad Med. 2018; 64(4): 247-249.

3. Gao M, Yang C, Wang X, et al. ApoC2 deficiency elicits severe hypertriglyceridemia and spontaneous atherosclerosis: A rodent model rescued from neonatal death. Metabolism. 2020; 109: 154296.

4. Siafakas CG, Brown MR, Miller TL. Neonatal pancreatitis associated with familial lipoprotein lipase deficiency. J Pediatr Gastroenterol Nutr. 1999; 29(1): 95-98.

5. Yıldız Y, Uysal Yazıcı M, Çınar HG, Özbay Hoşnut F, Kurt Çolak F, Kılıç M. Successful management of acute pancreatitis due to apolipoprotein C-II deficiency in a 37-day-old infant. Pancreatology. 2020; 20(4): 644-646.

6. Chou JY, Mansfield BC. Mutations in the glucose6-phosphatase-alpha (G6PC) gene that cause type Ia glycogen storage disease. Hum Mutat. 2008; 29(7): 921-930.

7. Talente GM, Coleman RA, Alter C, Baker L, Brown BI, Cannon RA, Chen YT, Crigler JF Jr, Ferreira P, Haworth JC, Herman GE, Issenman RM, Keating JP, Linde R, Roe TF, Senior B, Wolfsdorf JI: Glycogen storage disease in adults. Ann Intern Med. 1994; 120(4): 218-226.

8. Bandsma RH, Prinsen BH, van Der Velden Mde S, Rake JP, Boer T, Smit GP, Reijngoud DJ, Kuipers F: Increased de novo lipogenesis and delayed conversion of large VLDL into intermediate density lipoprotein particles contribute to hyperlipidemia in glycogen storage disease type 1a. Pediatr Res. 2008; 63(6): 702-707.

9. Carvalho PMS, Silva NJM, Dias PGD, Porto JFC, Santos LC, Costa JMN. Glycogen Storage Disease type 1a - a secondary cause for hyperlipidemia: report of five cases. J Diabetes Metab Disord. 2013; 12(1): 25.

10. Koeberl DD, Kishnani PS, Bali D, Chen Y-T. Emerging therapies for glycogen storage disease type I. Trends Endocrinol Metab. 2009; 20(5): 252-258. 
